\title{
Wild Animals Used as Food Medicine in Brazil
}

\author{
Rômulo Romeu Nóbrega Alves, 1 Tacyana Pereira Ribeiro Oliveira, ${ }^{2,3}$ \\ and Ierecê Lucena Rosa ${ }^{3}$ \\ ${ }^{1}$ Departamento de Biologia, Universidade Estadual da Paraíba, Avenida das Baraúnas, 351/Campus Universitário, \\ Bodocongó 58109-753, Campina Grande, PB, Brazil \\ ${ }^{2}$ Centro de Ciências Biológicas e Sociais Aplicadas, Universidade Estadual da Paraíba, Rua Horácio Trajano de Oliveira, s/n, \\ Campus V, 58020-540 João Pessoa, PB, Brazil \\ ${ }^{3}$ Departamento de Sistemática e Ecologia, Universidade Federal da Paraíba, 58059-900 João Pessoa, PB, Brazil
}

Correspondence should be addressed to Rômulo Romeu Nóbrega Alves; romulo_nobrega@yahoo.com.br

Received 5 April 2013; Accepted 4 July 2013

Academic Editor: Maria Franco Trindade Medeiros

Copyright (C) 2013 Rômulo Romeu Nóbrega Alves et al. This is an open access article distributed under the Creative Commons Attribution License, which permits unrestricted use, distribution, and reproduction in any medium, provided the original work is properly cited.

\begin{abstract}
The connection between eating and healing is common in traditional folk medical systems, and the multiple possibilities resulting from the combination of biodiversity and culture confer a wealth and complexity in terms of knowledge of the flora and fauna as to their potential as food medicine. The growing awareness of the links between traditional therapeutic-alimentary uses of wildlife and conservation has drawn attention to the gaps in knowledge on the social, economic, and biological contexts in which different forms of traditional wildlife uses take place, particularly with regard to zootherapeutic resources. In this study we interviewed 124 merchants and 203 traditional users of animal-derived remedies in Brazil, aiming at documenting the animal species used as foods and medicines in urban and rural areas of the country. At least 354 wild animal species are used in Brazilian traditional medicine, of which 157 are also used as food. The high degree of overlap between medicinal and alimentary uses of wild animals highlights the importance of understanding the socioeconomic, cultural, and ecological contexts in which those traditional uses take place for elucidating their potential impact on public health and biodiversity conservation.
\end{abstract}

\section{Introduction}

Nature-based traditional food and medicine are generally viewed as interchangeable, diet being highly regarded as the primary basis for sustaining and/or restoring health and wellbeing. Consequently, foods are considered and often times chosen for their distinctive medicinal or healing values [1-4]. For many traditional societies, now and in the past, food isat least partially-medicine, and medicine is food [5]. Medicinal cuisines and consumption of health edibles have held a central position in traditional folk medical systems [6-10].

Much of the plant material that is consumed by animals in the wild contains an array of secondary compounds. Johns [6] argues that the herbal medicines and modern pharmaceuticals used by humans today have replaced the nonnutritive chemicals commonly present in our primate ancestors' diets, the connection between eating and healing likely being as old as the use of biodiversity by humans [6]. For ancient Assyrians, Greeks, and Chinese, cultivated grains, roots, fruits, and spices were frequently employed in the curing arts, while, in pharaonic Egypt, plants such as lettuce, sesame, onions, leeks, cucumbers, plums, watermelon, and many other edibles were included in the healer's arsenal [11]. Historical sources of ancient Egypt also mention the medicinal uses of animal-derived substances, such as cattle milk, bee honey, lizard blood, ox organs, swallow's liver, bat limbs, ambergris from the sperm whale, and the glands of the musk deer [1215].

At the dawn of recorded history it is known that man often ate or wore on his person some portion of an animal that was thought to have a healing or protecting influence [16], and this aspect highlights that the origin of the medicinal use of faunal elements is intertwined with their use as food. In this same direction, Chemas [17] remarked that the treatment 
of illnesses using animal-based remedies is an extremely old practice, whose most remote ancestor is a carnivore diet, closely followed by the ritual ingestion of deceased persons (e.g., close relatives, warriors) as a means to absorb their virtues (e.g., courage, virility) and subsequently by a true medicinal use indissociable from magic-religious elements.

Since ancient times, the thinking of "food as medicine" has existed in Chinese medical theories and Chinese food therapy $[18,19]$. Books on Chinese cooking often emphasize the medicinal value of foods and the importance of "nutritional therapies" dating from earliest times, and many of the vegetable and animal products decocted in Chinese medicines are used routinely in cooking. During the 1980s, talk at banquets frequently revolved around the healthful properties of foods being consumed, and nutritional and food preparation advice was commonly tendered in clinics along with herbal prescriptions [20].

Research in several regions of the world has illustrated that many wild plants retained in local food cultures are inseparable from traditional therapeutic systems $[7,9,21$, 22]. For example, in a rural Hausa community in northern Nigeria, of the 119 plants identified as food, all but five are included among the total 374 medicinals. This, however, does not mean that Hausa intermixes the domains of food and medicine [7]. Examples of a number of food animals also used as remedies can be found in the literature [23-28]. Yet our knowledge about the practice of food medicine is limited, particularly with regard to the traditional consumption of animal food medicines [29].

Although often regarded as supplementary to local peoples' diet, wild food and medicine are essential in times of crisis and play an important nutritional role. Hence, the neglect of traditional food and medicines may seriously deteriorate the health and well-being of traditional peoples [30, 31]. Further, nature-based traditional food and medicine are generally viewed as interchangeable, diet being highly regarded as the primary basis for sustaining and/or restoring health and well-being. Consequently, foods are considered and often times chosen for their distinctive medicinal or healing values.

Animal medicinal foods have been broadly used since ancient times and have played a significant role in healing practices in Brazil [3, 32], where elements of indigenous, European, and African cultures met and produced a singular repertoire of species that are used as food and often also as medicine.

Brazil provides an interesting setting for several reasons: (a) the country possesses between 15 and $20 \%$ of all the world's biological diversity, as well as a significant cultural diversity, represented by more than 200 indigenous groups and by a large number of local communities which detain a considerable knowledge of the flora and fauna and of traditional systems of renewable natural resources management [25]; (b) the multiple possibilities resulting from this combination of biodiversity and culture confer a wealth and complexity in terms of knowledge of the Brazilian flora and fauna as to its therapeutic potential; (c) in addition, Brazil is vast, with parts of the territory of difficult access; this precludes some local populations from accessing services provided by the government's health care network. In many cases this geographical isolation contributes to strengthen traditional and local medical practices and, also, to prompt selection of natural resources for the treatment of new diseases [25, 33]. Interest in animal-derived remedies, however, extends beyond people lacking access to medical services in Brazil. As shown by Alves and Rosa [26, 27], even in cities where such services are more accessible, many people still resort to traditional healers, showing the cultural acceptability of such practices.

In this study we explored the medicinal and alimentary uses of wildlife in Brazil, aiming to (1) document the animal species used and the illnesses to which they were prescribed and (2) to discuss resource use in a conservationist context.

\section{Methods}

Data were collected from January 2002 to June 2012. Data consisted in (a) interviews in markets/shops located in the cities of Belém (Pará State), São Luís (Maranhão State), Teresina (Piauí State), Goiânia (Goiás State), Natal (Rio Grande do Norte State), João Pessoa, Campina Grande (Paraíba State), Recife (Pernambuco State), Maceió (Alagoas State), Aracajú (Sergipe State), Salvador (Bahia State), Vitória (Espírito Santo State), Niterói (Rio de Janeiro State), Florianópolis (Santa Catarina State), and Porto Alegre (Rio Grande do Sul State), where we documented the animal medicinal foods traded, (b) interviews in outdoor markets to 124 merchants about the use and commercialization of medicinal animals (23 interviewees in Belém, 21 in São Luís, 21 in Teresina, 16 in Santa Cruz, 11 in Caruaru, 10 in João Pessoa, and 22 in Campina Grande), (c), and interviews with 203 traditional users of animal-derived remedies (67 men and 70 women) in the following rural communities: Municipality of Cajueiro da Praia (Piauí State) $(n=36)$, Pesqueiro Beach, Municipality of Soure (Pará State) $(n=41)$, Environmental Protected Area Barra do Rio Mamanguape, Municipality of Rio Tinto (Paraíba State) $(n=30)$, Municipality of Queimadas (Paraíba State) $(n=66)$, and Municipality of Raposa (Maranhão State) $(n=30)$, as described in Alves and Rosa $[1,25,26]$.

In cities, the sampling method was nonrandom, and the interviewees were predefined [34]. Despite attempts to interview all animal merchants in the markets visited, some interviews were cancelled. Others proved to be fruitless, because interviewees were reluctant to answer questions. At the surveyed fishing communities, we identified local people with a specialized knowledge of medicinal animal use. Additional interviewees were chosen by using the snowball technique [35], based on information initially provided by the specialists.

To respect intellectual property rights, we adopted the following protocol in the field: before the survey, we introduced ourselves, explained the nature and objectives of our research, and asked the respondents for permission to record the information. The ethical approval for the study was obtained from the Ethics Committee of Paraiba University State.

The information obtained through semistructured interviews was complemented by free interviews [36], and, 
for each animal cited, respondents were requested to furnish vernacular name, folk use, parts used, preparation and administration of remedy, and which animal species are also used as food. Zoological material was identified with the aid of specialists, through (1) examination of voucher specimens (donated by the interviewees) (2) photographs of the animals or their parts, taken during interviews; and (3) vernacular names, with the aid of taxonomists familiar with the study areas' fauna. Only wild animals and taxa that could be identified to species level were included in the database.

Records of animal-based folk remedies were gathered from scientific articles, books, and book chapters, theses, and dissertations, as well as from reports available in international online databases such as Science Direct (http://www.sciencedirect.com/), Scirus (http://www.scirus .com/), Google Scholar, Scopus (http://www.scopus.com/), Web of Science (http://www.isiknowledge.com/), and Biological Abstracts (http://science.thomsonreuters.com/) using the following search terms: medicinal animals + use + Brazil, zootherapy + commercialization + Brazil, hunting + medicinal animals + Brazil, and, and zootherapy + Brazil.

Whenever applicable, scientific names provided in publications were updated using ITIS Catalogue of Life: 2012 Annual Checklist (http://www.catalogueoflife.org/).

Information on the conservation status of animal species was obtained from the International Union for Conservation of Nature's Red List (http://www.iucnredlist.org/), the Convention on the International Trade in Endangered Species of Wild Fauna and Flora (http://www.cites.org/eng/ resources/species.html), Brazil's Official List of Endangered Species [37] and National List of Species of Aquatic Invertebrates and Fishes Endangered, Overexploited, or Threatened of Exploitation [38].

\section{Results and Discussion}

Animals used as medicine food recorded in our study were distributed in six zoological groups. As shown in Figure 1, the taxon with the largest number of species was fishes (77 species; $49.0 \%)$, followed by mammals $(35 ; 22.3 \%)$ and reptiles $(20 ; 12.7 \%)$. These results are in line with previous studies carried out elsewhere (eg., 28, 40, and 4146), further highlighting the widespread use of wild-caught vertebrates in the diets and medical systems of different societies.

Generally, species are harvested through fishing or hunting, mainly for alimentary purposes. In this sense, their utilization as remedies potentializes resource use. Meat, the principal product consumed as food, in some cases was also consumed due to a perceived medicinal value. This distinction, however, was not always clear-cut, as the same animal can be one, the other, or both categories at the same time, depending on the parts used, the method of preparation, and the state of health or pathology of the individual being treated. This result is in line with Huffman [39], who remarked that in traditional human societies, the difference between food and medicine may not always be clear. In fact, according to O'Hara-May [40], the beginnings

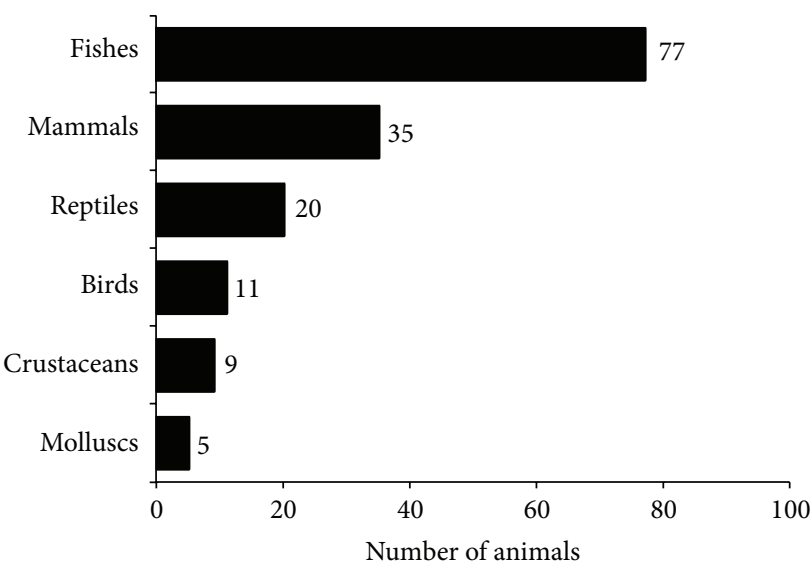

FIgURE 1: Number of animal species, per taxonomic category, used as food and medicines in Brazil.

of the medicinal uses of animals in human history are clear: animals and their products were part of the primary resources that ancient peoples could use as food or for treating their illnesses.

Most of the time, the hunted or fished animal whose meat is consumed as food also provides byproducts that are used for medicinal purposes, such as skin or fats. In fact, the utilization of remainings or by-products seems to be widespread and one of the most striking characteristics of the Brazilian folk medicine, in terms of medicinal animals [41]. In this sense, it is remarkable that in general the animal-based medicinal products constitute by-products from animals hunted for other purposes. Such multiple uses (including medicinal) of fauna and their impact on animal populations must be properly assessed and taken into consideration when implementing recovery plans for these target species, especially those that are highly exploited [3, 27, 42].

The high number of fish species recorded as medicinal foods in this study was expected, given their high consumption as food, mainly in coastal areas. As pointed by Burger and Gochfeld [43], in many parts of the world more than half of the people live in coastal communities where fish is prominent in their diets. Although people who live near the sea eat more seafood than those who live in the hinterland, seafood, both fresh and frozen, has become increasingly available and is gaining in popularity throughout the world. In addition to being an important and available source of protein, the popularity of fish as food is also due to the fact that they are considered healthy. Fishes are considered an excellent and low-fat source of protein, provide many health benefits, such as omega-3 fatty acids that reduce cholesterol levels and the incidence of stroke, heart disease, and preterm delivery, and enhance cognitive development [44-52].

Among terrestrial vertebrates, mammals are the most hunted taxon for alimentary purposes. The frequent use of those animals as medicinal foods was expected, given their comparatively larger body size (when compared with other terrestrial vertebrates) and the possibility they offer of a higher energetic intake. In the neotropical region, mammals 
TABLE 1: Animal species used as food and medicine in Brazil and conditions to which remedies are prescribed.

\begin{tabular}{|c|c|}
\hline Taxonomic category/species & Conditions to which remedies are prescribed \\
\hline \multicolumn{2}{|l|}{ Molluscs } \\
\hline Phacoides pectinata (Gmelin, 1791) & Sexual impotence \\
\hline Mytella charruana (Orbigny, 1842) & Ophthalmological problems \\
\hline Mytella guyanensis (Lamarck, 1819) & Weakness \\
\hline Crassostrea rhizophorae (Guilding, 1828) & $\begin{array}{l}\text { Osteoporosis, pneumonia, stomach ache, cancer, flu, } \\
\text { weakness, pain relief in injuries caused by the dorsal fin } \\
\text { spine of a species of catfish, anemia, and tuberculosis }\end{array}$ \\
\hline Anomalocardia brasiliana (Gmelin, 1791) & Asthma, flu, stomach and ache \\
\hline \multicolumn{2}{|l|}{ Crustaceans } \\
\hline Cardisoma guanhumi (Latreille, 1825) ${ }^{\mathrm{NE} / \mathrm{IN}}$ & Asthma, bronchitis, wounds, and boils \\
\hline Goniopsis cruentata (Latreille, 1802) & Epilepsy, venereal disease \\
\hline Ucides cordatus (Linnaeus, 1763) & $\begin{array}{l}\text { Hemorrhage in women, incontinence, osteoporosis, } \\
\text { cough, asthma, tuberculosis, womb disorders, arthrosis, } \\
\text { and bronchitis }\end{array}$ \\
\hline Macrobrachium carcinus (Linnaeus, 1758) & Amnesia \\
\hline Macrobrachium acanthurus (Wiegmann, 1836) & Irritation when milk teeth are erupting \\
\hline Macrobrachium borellii (Nobili, 1896) & Irritation when milk teeth are erupting \\
\hline Xiphopenaeus schmitti (Burkenroad, 1936) & Irritation when milk teeth are erupting, skin spots \\
\hline Xiphopenaeus kroyeri (Heller, 1862) & Irritation when milk teeth are erupting, skin spots \\
\hline Aratus pisonii (H. Milne Edwards, 1837) & $\begin{array}{l}\text { Epilepsy, to alleviate the symptoms of intoxication with } \\
\text { poison of Colomesus psittacus (a species of pufferfish) }\end{array}$ \\
\hline \multicolumn{2}{|l|}{ Fishes } \\
\hline Trachelyopterus galeatus (Linnaeus, 1766) & Umbilical hernia, asthma, and sexual impotence \\
\hline Leporinus friderici (Bloch, 1794) & Earache \\
\hline Schizodon knerii (Steindachner, 1875) & Leucoma, edema \\
\hline Bagre bagre (Linnaeus, 1766 ) & Injuries caused by itself \\
\hline Genidens barbus (Lacepède, 1803) & Pain relief caused in injuries by the species' sting \\
\hline Genidens genidens (Cuvier, 1829) & Injuries caused by itself \\
\hline Sciadeichthys luniscutis (Valenciennes, 1837) & Pain relief caused in injuries by the species' sting \\
\hline Aspredo aspredo (Linnaeus, 1758) & Asthma \\
\hline Aspredinichthys tibicen (Valenciennes, 1840) & Asthma \\
\hline Balistes capriscus (Gronow, 1854) ${ }^{\mathrm{DD} / \mathrm{IN}}$ & Bronchitis \\
\hline Balistes vetula (Linnaeus, 1758$)^{\mathrm{VU}}$ & $\begin{array}{l}\text { Stroke, asthma, thrombosis, earache, pain relief caused } \\
\text { in injuries by the species' sting, hemorrhage, ascites, } \\
\text { schistosomiasis, appendicitis, menstrual cramps, and } \\
\text { gastritis }\end{array}$ \\
\hline Thalassophryne nattereri (Steindachner, 1876) & Pain relief caused in injuries by the species' sting \\
\hline Callichthys callichthys (Linnaeus, 1758) & Asthma, umbilical hernia \\
\hline Carcharhinus limbatus (Müller and Henle, 1839) ${ }^{\mathrm{LC}}$ & Osteoporosis \\
\hline Carcharhinus porosus (Ranzani, 1840) & $\begin{array}{l}\text { Asthma, rheumatism, wounds, inflammations, } \\
\text { osteoporosis, and anemia }\end{array}$ \\
\hline Galeocerdo cuvier (Péron and Lesueur, 1822) & Osteoporosis \\
\hline Rhizoprionodon lalandii (Müller and Henle, 1839) & Rheumatism \\
\hline Rhizoprionodon porosus (Poey, 1861) & Rheumatism \\
\hline Sphyrna lewini (Griffith and Smith, 1834) LC/IN $^{\mathrm{L}}$ & Asthma, wounds, rheumatism, and inflammation \\
\hline Centropomus parallelus (Poey, 1860) & Nephritis \\
\hline Centropomus undecimalis (Bloch, 1792) & Edema in the legs \\
\hline Astyanax bimaculatus (Linnaeus, 1758) & $\begin{array}{l}\text { Alcoholism, leishmaniasis, skin burns, wounds, and } \\
\text { rheumatism }\end{array}$ \\
\hline Brycon nattereri (Günther, 1864) & Flu \\
\hline Colossoma macropomum (Cuvier, 1818) DD/IN $^{\mathrm{D}}$ & Paralysi of arms and legs \\
\hline
\end{tabular}


TABle 1: Continued.

\begin{tabular}{|c|c|}
\hline Taxonomic category/species & Conditions to which remedies are prescribed \\
\hline Hydrolycus scomberoides (Cuvier, 1816) & Earache \\
\hline Opisthonema oglinum (Lesueur, 1818) & Alcoholism \\
\hline Dasyatis guttata (Bloch and Schneider, 1801) & $\begin{array}{l}\text { Asthma, pain relief caused in injuries by the species' } \\
\text { sting, and burns }\end{array}$ \\
\hline Dasyatis marianae (Gomes, Rosa, and Gadig, 2000) & $\begin{array}{l}\text { Asthma, pain relief caused in injuries by the species' } \\
\text { sting, and burns }\end{array}$ \\
\hline Franciscodoras marmoratus (Reinhardt, 1874) & Injuries caused by itself \\
\hline Lithodoras dorsalis (Valenciennes, 1840) & Swelling \\
\hline Megalodoras uranoscopus (Eigenmann and Eigenmann, 1888) & Rheumatism \\
\hline Platydoras costatus (Linnaeus, 1758) & Rheumatism \\
\hline Pterodoras granulosus (Valenciennes, 1821) & Rheumatism \\
\hline Oxydoras niger (Valenciennes, 1821) & Rheumatism \\
\hline Echeneis naucrates Linnaeus, 1758 & Asthma, bronchitis \\
\hline Erythrinus erythrinus (Bloch and Schneider, 1801) & Asthma \\
\hline Hoplias malabaricus (Bloch, 1794) & $\begin{array}{l}\text { Ophthalmological problems, rheumatism, cataracts, } \\
\text { wounds, snake bite, conjunctivitis, stroke, thrombosis, } \\
\text { asthma, toothache, fever, earache, diarrhea, deafness, } \\
\text { boils, bleedings, alcoholism, tetanus, sore throat, } \\
\text { itching, sprains, and leucoma }\end{array}$ \\
\hline Gadus morhua (Linnaeus, 1758) ${ }^{\mathrm{VU}}$ & Boils \\
\hline Ginglymostoma cirratum (Bonnaterre, 1788$)^{\mathrm{DD}}$ & Rheumatism \\
\hline Pimelodella brasiliensis (Steindachner, 1876) & Injuries caused by that fish species \\
\hline Holocentrus adscensionis (Osbeck, 1765) & Wounds \\
\hline Megalops atlanticus (Valenciennes, 1847) & $\begin{array}{l}\text { Stroke, headache, asthma, shortness of breath, } \\
\text { thrombosis, chest pain, and injuries caused by bang }\end{array}$ \\
\hline Gymnothorax funebris (Ranzani, 1840) & Bleeding (wounds) \\
\hline Gymnothorax moringa (Cuvier, 1829) & Bleeding (wounds) \\
\hline Gymnothorax vicinus (Castelnau, 1855) & Bleeding (wounds) \\
\hline Aetobatus narinari (Euphrasen, 1790) ${ }^{\mathrm{LC}}$ & $\begin{array}{l}\text { Asthma, pain relief caused in injuries by the species' } \\
\text { sting, burns, and hemorrhage }\end{array}$ \\
\hline Narcine brasiliensis (Olfers, 1831) & Toothache \\
\hline Arapaima gigas (Schinz, 1822) & Asthma, pneumonia \\
\hline Phractocephalus hemioliopterus (Bloch and Schneider, 1801) & $\begin{array}{l}\text { Asthma, wounds, hernia, burns in the skin, } \\
\text { rheumatism, flu, and cough }\end{array}$ \\
\hline Pseudoplatystoma corruscans (Spix and Agassiz, 1829) & Flu \\
\hline Pseudoplatystoma fasciatum (Lunnaeus, 1776) & Cold \\
\hline Sorubimichthys planiceps (Spix and Agassiz, 1829) & Leishmaniasis, tuberculosis \\
\hline Zungaro zungaro (Humboldt, 1821) $\mathrm{DD} / \mathrm{IN}$ & $\begin{array}{l}\text { Asthma, toothache, earache, wounds, athlete's foot, } \\
\text { burns in the skin, rheumatism, and flu }\end{array}$ \\
\hline Paratrygon aiereba (Müller and Henle, 1841) & $\begin{array}{l}\text { Asthma, hernia, flu, pneumonia, cough, earache, and } \\
\text { burns }\end{array}$ \\
\hline Potamotrygon hystrix (Müller and Henle, 1834) & $\begin{array}{l}\text { Asthma, hernia, flu, pneumonia, cough, earache, and } \\
\text { burns }\end{array}$ \\
\hline Potamotrygon motoro (Müller and Henle, 1841) & $\begin{array}{l}\text { Asthma, hernia, flu, pneumonia, cough, earache, and } \\
\text { burns }\end{array}$ \\
\hline Potamotrygon orbignyi (Castelnau, 1855) & Pain relief caused in injuries by that species' sting \\
\hline Plesiotrygon iwamae (Rosa, Castello, and Thorson, 1987) & $\begin{array}{l}\text { Pain relief caused in injuries by the species' sting, } \\
\text { wounds, and cracks in the sole of the feet }\end{array}$ \\
\hline Pristis pectinata (Latham, 1794$)^{\mathrm{CR}}$ & Asthma, rheumatism, and arthritis \\
\hline Pristis perotteti (Müller and Henle, 1841) ${ }^{\mathrm{CR}}$ & Asthma, rheumatism, and arthritis \\
\hline Prochilodus argenteus (Spix and Agassiz, 1829) & To avoid swelling of the breast feeding, mycosis \\
\hline Prochilodus nigricans (Spix and Agassiz, 1829) & $\begin{array}{l}\text { Chilblain, skin burns, wounds, rheumatism, and eye } \\
\text { pains }\end{array}$ \\
\hline
\end{tabular}


TABle 1: Continued.

\begin{tabular}{ll}
\hline Taxonomic category/species & Conditions to which remedies are prescribed \\
\hline Atlantoraja cyclophora (Regan, 1903) $\mathrm{VU}$ & Hemorrhage after delivery \\
Mylossoma duriventre (Cuvier, 1818) & Venereal disease \\
Serrasalmus brandtii (Lütken, 1875) & Inflammations, sexual impotence \\
Cynoscion acoupa (Lacepède, 1801) & Renal failure \\
Cynoscion leiarchus (Cuvier, 1830) & Renal failure \\
Micropogonias furnieri (Desmarest, 1823) & Pain relief caused in injuries by the species' sting, \\
Pachyurus francisci (Cuvier, 1830) & cough, asthma, and bronchitis \\
Plagioscion surinamensis (Bleeker, 1873) & Asthma, urinary incontinence, and backache \\
Plagioscion squamosissimus (Heckel, 1840) & Urinary disorders, hemorrhage, and snake bites \\
Calamus penna (Valenciennes, 1830) & Urinary disorders, hemorrhage, and snake bites \\
Synbranchus marmoratus (Bloch, 1795) & Asthma \\
Colomesus psittacus (Bloch and Schneider, 1801) & Bronchitis \\
Sphoeroides testudineus (Linnaeus, 1758) & Breast cancer, backache, and warts \\
Trichiurus lepturus (Linnaeus, 1758) & Rheumatism \\
Urotrygon microphthalmum (Delsman, 1941) & Asthma \\
\hline
\end{tabular}

Reptiles

Iguana iguana (Linnaeus, 1758$)^{\mathrm{DD} / \mathrm{II}}$

Tupinambis merianae (Duméril and Bibron, 1839) DD/II $^{\mathrm{D}}$

Tupinambis teguixin (Linnaeus 1758) DD/II $^{\mathrm{D}}$

Boa constrictor (Linnaeus, 1758) $)^{\mathrm{DD} / \mathrm{II}}$

Eunectes murinus (Linnaeus, 1758) ${ }^{\mathrm{DD} / \mathrm{II}}$

Caretta caretta (Linnaeus, 1758) $\mathrm{VU/I}$

Chelonia mydas (Linnaeus, 1758) $)^{\mathrm{VU} / \mathrm{I}}$
Earache, erysipelas, asthma, rheumatism, edema, abscesses, joint pain, wounds, acne, athlete's foot, sore throat, swelling, burn, tumor, to suck a splinter out of skin or flesh, boil, injuries caused by the spines of the "arraia" and others fishes, inflammation, and hernia Earache, deafness, rheumatism, erysipelas, skin thorns and wounds, respiratory diseases, sore throat, snake bite, asthma, tumor, swelling, infection, and bronchitis

Sexual impotence, rheumatism, erysipelas, dermatitis, snake bites, asthma, tetanus, earache, thrombosis, wounds, paronychia, swelling, herpes zoster, irritation when milk teeth are erupting, jaundice, inflammation, tumor, sore throat, infection, bronchitis, injuries caused by the spines of the "arraia," pain relief in injuries caused by snake bites, toothache, suck a splinter out of skin or fresh, headache, cough, stroke, and coarse throat Rheumatism, lung disease, thrombosis, boils, tuberculosis, stomach ache, edema, snake bite, cancer, ache, swelling, to prevent abort, pain in the body, inflammation, athlete's foot, calluses, tumors, cracks in the sole of the feet, goiter, sore throat, arthrosis, insect sting, dog bite, erysipelas, thrombosis, asthma, neck strain, and strain muscle

Wounds, skin problems, bruises, sprains, arthrosis, rheumatism, boils, sexual impotence, headache, sore throat, thrombosis, swelling, tumour, asthma, muscle strain, numbness, syphilis, to reduce pain, and luxation Injuries caused by bang, toothache, diabetes, headache, backache, wounds, cough, bronchitis, asthma, thrombosis, rheumatism, stroke, hoarseness, flu, backache, earache, sore throat, and swelling Injuries caused by bang, toothache, diabetes, headache, backache, wounds, cough, bronchitis, asthma, flu, thrombosis, rheumatism, toothache, stroke, hoarseness, earache, sore throat, swelling, whooping cough, arthritis, erysipelas, boil, wounds, arthrosis, and inflammation 
TABLE 1: Continued.

Taxonomic category/species

Eretmochelys imbricata (Linnaeus, 1766) ${ }^{\mathrm{EN} / \mathrm{I}}$

Lepidochelys olivacea (Eschscholtz, 1829) ${ }^{\mathrm{EM} / \mathrm{I}}$

Dermochelys coriacea (Vandelli, 1761) ${ }^{\mathrm{CR} / \mathrm{I}}$

Rhinoclemmys punctularia (Daudin, 1802)

Podocnemis expansa (Schweiger, 1812) ${ }^{\mathrm{LC} / \mathrm{II}}$

Podocnemis unifilis (Troschel, 1848) VU/II

Podocnemis sextuberculata (Cornalia, 1849)

Peltocephalus dumerilianus (Schweigger, 1812)

Caiman crocodilus (Linnaeus, 1758) LC/II $^{\mathrm{L}}$

Caiman latirostris (Daudin, 1801) ${ }^{\mathrm{LC} / \mathrm{II}}$

Melanosuchus niger (Spix, 1825) ${ }^{\mathrm{LC} / \mathrm{II}}$

Paleosuchus palpebrosus (Cuvier, 1807) ${ }^{\mathrm{LC} / \mathrm{II}}$

Paleosuchus trigonatus (Schneider, 1801) ${ }^{\mathrm{DD} / \mathrm{II}}$

Birds

Anser anser (Linnaeus, 1758)

Ardea cocoi (Linnaeus, 1766)

Penelope jacucaca $\left(\right.$ Spix, 1825) ${ }^{\mathrm{VU}}$

Ciconia maguari (Gmelin, 1789)

Leptotila rufaxilla (Richard and Bernard, 1792)

Columba livia (Gmelin, 1789)

Meleagris gallopavo (Linnaeus, 1758)

Rhea americana (Linnaeus, 1758) ${ }^{\mathrm{LC} / \mathrm{II}}$

Crypturellus noctivagus (Wied, 1820) $\mathrm{vU}$

Nothura boraquira (Spix, 1825)

Rhynchotus rufescens (Temminck, 1815)
Conditions to which remedies are prescribed

Injuries caused by bang, toothache, diabetes, headache, backache, wounds, cough, bronchitis, asthma, thrombosis, stroke, hoarseness, flu, rheumatism, earache, sore throat, and swelling

Injuries caused by bang, toothache, diabetes, headache, backache, wounds, cough, flu, bronchitis, asthma, thrombosis, rheumatism, stroke, and hoarseness

Rheumatism, earache, sore throat, and swelling Wounds, tumor, erysipelas, earache, and rheumatism Inflammation, acne, tumor, boil, rheumatism, pterygium, skin spots, backache, earache, arthrosis, arthritis, swelling, and wrinkle

Wounds, tumor, erysipelas, earache, and rheumatism

Blackhead; acne

Blackhead; acne

Asthma, stroke, bronchitis, backache, earache, rheumatism, thrombosis, sexual impotence, snake bites (antidote), evil eye, irritation when milk teeth are erupting, discharge, swelling, scratch, athlete's foot, ophthalmological problems, asthma, sore throat, amulet used as a protection against snake bite, rheumatism, hernia, prostate problems, infection, and thrombosis Asthma, sore throat, amulet used as a protection against snake bite, rheumatism, irritation when milk teeth are erupting, hernia, and prostate problems

Thrombosis, infection, swelling, asthma, amulet used as a protection against snake bite, injuries caused by spines of the "arraia," and pain relief in injuries caused by snake bites

Snake bite, asthma, stroke, rheumatism, thrombosis, backache, sexual impotence, edema, mycosis, evil eye, irritation when milk teeth are erupting, snake bite (antidote), discharge, sore throat, amulet used as a protection against snake bite, hernia, and prostate problems

Rheumatism

Laryngitis, pharyngitis, and tonsillitis

Swelling, inflammation, injuries caused by the spines of the "arraia" and others fishes, asthma, boil, tumor, inflammation, rheumatism, and earache

Insomnia

Injuries caused by the spines of the "arraia" and others fishes, and thrombosis

Thrombosis

Asthma, laryngitis, pharyngitis, and tonsillitis

Asthma

General aches, rheumatism, thrombosis, and strokes

Thrombosis, stroke

Thrombosis, stroke

Snake bite, thrombosis, and snake bites (antidote) 
TABle 1: Continued.

\begin{tabular}{|c|c|}
\hline Taxonomic category/species & Conditions to which remedies are prescribed \\
\hline \multicolumn{2}{|l|}{ Mammals } \\
\hline Agouti paca (Linnaeus, 1766) ${ }^{\mathrm{LC} / I I I}$ & $\begin{array}{l}\text { Wound in the breast caused by suckling, } \\
\text { ophthalmological problems, stomach disorders, } \\
\text { pterygium, to suck a splinter out of skin or flesh, } \\
\text { injuries caused by the spines of "arraia," and control } \\
\text { cholesterol level }\end{array}$ \\
\hline Bubalus bubalis (Linnaeus, 1758) & Rheumatism, osteoporosis, and thrombosis \\
\hline Capra hircus (Linnaeus, 1758) & Evil eye, snake bite, and muscle strain \\
\hline Bradypus variegatus (Shinz, 1825) & Thrombosis \\
\hline Bradypus tridactylus (Linnaeus, 1758) & Thrombosis, insects bite, and scorpions bite \\
\hline Cavia aperea (Erxleben, 1777) & Inflammation \\
\hline Kerodon rupestris (Wied-Neuwied, 1820) & Constipation \\
\hline Alouatta belzebul (Linnaeus, 1766) ${ }^{\mathrm{CR}}$ & Whooping cough, sore throat, and asthma \\
\hline Alouatta nigerrima (Lönnberg, 1941) & Whooping cough, inflammation \\
\hline Alouatta seniculus (Linnaeus, 1766) ${ }^{\mathrm{LC} / \mathrm{II}}$ & $\begin{array}{l}\text { Whooping cough, inflammation, and to accelerate } \\
\text { parturition }\end{array}$ \\
\hline Cebus apella (Linnaeus, 1758) (L/II $^{\mathrm{L}}$ & Insect sting \\
\hline Blastocerus dichotomus (Illiger, 1815) $\mathrm{VU} / \mathrm{I}$ & Diarrhea, vomit \\
\hline Mazama americana (Erxleben, 1777$)^{\mathrm{DD} / \mathrm{III}}$ & Stroke \\
\hline Mazama simplicicornis (Illinger, 1811) & Diarrhea, verminosis, and evil eye \\
\hline Mazama cf. gouazoubira (G. Fischer, 1814) & $\begin{array}{l}\text { Asthma, edema, rheumatism, snake bite, thrombosis, to } \\
\text { assist children who take longer than usual to start } \\
\text { walking, toothache, wounds, and sprains }\end{array}$ \\
\hline Ozotocerus bezoarticus (Linnaeus, 1758) & Diarrhea, verminosis, and evil eye \\
\hline Dasypus novemcinctus (Linnaeus, 1758) & $\begin{array}{l}\text { Thrombosis, insects bite, scorpions bite, edema, } \\
\text { asthma, deafness, earache, and evil eye }\end{array}$ \\
\hline Euphractus sexcinctus (Linnaeus, 1758) & $\begin{array}{l}\text { Wounds, earache, evil eye, asthma, sore throat, } \\
\text { pneumonia, sinusitis, deafness, and coarse throat }\end{array}$ \\
\hline Tolypeutes tricinctus (Linnaeus, 1758) $\mathrm{VU}$ & Thrombosis, rheumatism \\
\hline Dasyprocta prymnolopha (Wagler, 1831) & Asthma, thrombosis \\
\hline Sotalia fluviatilis (Gervais and Deville, 1853$)^{\mathrm{DD} / \mathrm{I}}$ & $\begin{array}{l}\text { Asthma, headache, rheumatism, hernia, womb } \\
\text { disorders, sore throat, injuries caused by the spines of } \\
\text { the "arraia," swelling, hemorrhoids inflammation, } \\
\text { wounds, earache, erysipelas, athlete's foot, tumor, and } \\
\text { cancer }\end{array}$ \\
\hline Sotalia guianensis (P. J. Van Bénéden, 1864) & $\begin{array}{l}\text { Asthma, headache, rheumatism, hernia, womb } \\
\text { disorders, sore throat, injuries caused by the spines of } \\
\text { the "arraia," swelling, hemorrhoids inflammation, } \\
\text { wounds, earache, erysipelas, athlete's foot, tumor, and } \\
\text { cancer }\end{array}$ \\
\hline Coendou prehensilis (Linnaeus, 1758) & $\begin{array}{l}\text { Bronchitis, thrombosis, epilepsy, stroke, abscesses, } \\
\text { conjunctivitis, and asthma }\end{array}$ \\
\hline Hydrochaeris hydrochaeris (Linnaeus, 1766) & $\begin{array}{l}\text { Thrombosis, conjunctivitis, venereal disease, } \\
\text { rheumatism, earache, strengthen bones, liver pain, } \\
\text { bronchitis, asthma, wounds, erysipelas, and cough }\end{array}$ \\
\hline Inia geoffrensis (Blainville, 1817) $\mathrm{VU/II}$ & $\begin{array}{l}\text { Asthma, headache, rheumatism, hernia, womb } \\
\text { disorders, sore throat, injuries caused by the spines of } \\
\text { the "arraia," swelling, hemorrhoids inflammation, } \\
\text { wounds, earache, erysipelas, athlete's foot, tumor, and } \\
\text { cancer }\end{array}$ \\
\hline Sylvilagus brasiliensis (Linnaeus, 1758) & Thrombosis, conjunctivitis, boils, and burns \\
\hline Conepatus semistriatus (Boddaert, 1785) & Rheumatism \\
\hline
\end{tabular}


TABLE 1: Continued.

\begin{tabular}{|c|c|}
\hline Taxonomic category/species & Conditions to which remedies are prescribed \\
\hline Lontra longicaudis (Olfers, 1818$)^{\mathrm{DD} / \mathrm{I}}$ & Thrombosis \\
\hline Myrmecophaga tridactyla (Linnaeus, 1758) & Thrombosis, stroke \\
\hline Myrmecophaga tetradactyla (Linnaeus, 1758) & Edema, thrombosis \\
\hline Tapirus terrestris (Linnaeus, 1758) $\mathrm{VU} / \mathrm{II}$ & $\begin{array}{l}\text { Rheumatism, arthrosis, osteoporosis, bursitis, muscular } \\
\text { pain, asthma, and tonsillitis }\end{array}$ \\
\hline Pecari tajacu (Linnaeus 1758$)^{\mathrm{LC} / I \mathrm{I} / \mathrm{III}}$ & Thrombosis, bronchitis, and stroke \\
\hline Tayassu pecari (Link, 1795) ${ }^{\mathrm{LC} / \mathrm{II}}$ & Thrombosis, stroke \\
\hline Trichechus inunguis (Natterer, 1883) & $\begin{array}{l}\text { Sprains, vaginal discharge, injuries caused by bang, } \\
\text { burns, asthma, menstrual cramps, rheumatism, sore } \\
\text { throat, wounds, muscle strain, suck a splinter out of } \\
\text { skin or fresh, tumor, backache, hernia, arthrosis, } \\
\text { luxation, menstrual cramps, and insects bite }\end{array}$ \\
\hline Trichechus manatus (Linnaeus, 1758) ${ }^{\mathrm{CR} / \mathrm{I}}$ & $\begin{array}{l}\text { Sprains, vaginal discharge, injuries caused by bang, } \\
\text { burns, asthma, menstrual cramps, rheumatism, sore } \\
\text { throat, wounds, muscle strain, suck a splinter out of } \\
\text { skin or fresh, tumor, backache hernia, arthrosis, } \\
\text { luxation, menstrual cramps, and insects bite }\end{array}$ \\
\hline
\end{tabular}

Categories of IUCN Red List: CR: critically endangered, EN: endangered, VU: vulnerable, LC: least concern, DD: data deficient, and NE: not evaluated. Cites appendices (I, II, and III); IN: Anexo 2-Instrução Normativa n. 5/2004/MMA.

clearly constitute the most important taxonomic group in terms of the number of species used by rural communities [53-60].

Reptiles ranked third in the number of species recorded used as food and medicine in this study and are among the animals most frequently used in folk medicine; the consumption of reptile meat is often intertwined with cultural or medicinal beliefs [61-67]. In this study, chelonians stood out as the reptiles most used as medicinal food ( $n=13$ species), a result in line with their extensive use as food in Brazil. As shown by Alves et al. [66], of the 36 species of chelonians in Brazil, 20 (55.5\%) are eaten by humans. Those animals are commonly sought after as food in the northern region of the country where they achieve the highest species richness and abundance. In a smaller proportion, lizards and caymans are also important as food medicine; on the other hand, only a few snake species have been used as food, despite the reported use of several species in Brazilian traditional medicine [64, 66]. The small number of snake species currently used as food in Brazil is not surprising given the negative images attributed to these animals in myths, legends, and popular beliefs [6668]. Rea [69] noted that not only snakes are rejected because of their disagreeable nature, but also any other creature with a similar shape or behavior will receive similar treatment. A study undertaken among human populations living along the banks of the Rio Negro River (Amazonas State, Brazil) showed that the electric eel (Electrophorus electricus) was one of the least favored meats because of its strong smell and the shape of its body-"it looks just like a snake" [70].

Some of the animals quoted by interviewees were mainly hunted for medicinal purposes, an example being the boa snake (Boa constrictor), which is eventually also used as food [66]. Conversely, other species are hunted for consumption, and their byproducts are utilized for medicinal purposes. At least 354 wild animal species are used in Brazilian traditional medicine [71], of which 157 (44.3\%) are also used as food (Table 1), a result that mirrors the central role played by wildlife as a source of protein in different parts of the world. As shown by previous studies, in at least 62 countries worldwide, wildlife (including fish) provides significant proteins, calories, and essential fats to rural communities [58-60, 7276]. It should be noted, however, that the number of animal species used as medicinal food in Brazil was higher than the number of species recorded for those purposes elsewhere (see [23]), possibly as a result of the country's significant biological and cultural diversity [77].

The overlap between alimentary and medicinal use can be exemplified by the use of caymans (Caiman latirostris, C. crocodilus, Paleosuchus palpebrosus, and Melanosuchus niger). While their meat was primarily consumed as food, their teeth, skin, fat, and penis were used for treating diseases such as asthma, stroke, bronchitis, backache, earache, rheumatism, thrombosis, sexual impotence, swelling, ophthalmological problems, sore throat, infection, thrombosis, swelling, injuries caused by spines of stingray, and pain relief in injuries caused by snake bites. Interestingly, caymans were also used as amulets to protect against snake bite or against evil eye [64, 65]. Likewise, the meat of armadillos (Euphractus sexcintus and Dasypus novemcinctus) was used as food, while their tail and skin were used for treating earache and asthma and as an amulet to protect against evil eye.

Another interface between the use of animals as food and medicine was expressed through the need, by those taking animal-based medicines, to control their diet-otherwise the medication would not work. Similar findings were described by Begossi [4] and Seixas and Begossi [78] who recorded the use of the word "carregado" to encompasses a set of supposed attributes of an animal (such as teeth, blood, 
aggressive behavior, "strong flesh," and fattiness) and factors that could provoke an inflammation if the animal was eaten by a wounded or unhealthy person.

Although the main part used for alimentary purposes was the flesh, the eggs and viscera of some species were also used. Examples include the Amazon River turtle Podocnemis expansa (Schweigger, 1812), the Black Vulture Coragyps atratus (Bechstein, 1793), the smooth-billed ani Crotophaga ani (Linnaeus, 1758), the red-footed tortoise Chelonoidis carbonaria (Spix, 1824), the yellow-footed tortoise Chelonoidis denticulata (Linnaeus, 1766), and the domestic chicken Gallus gallus (Linnaeus, 1758).

The consumption of the meat of reptiles, mammals, birds, and fishes is often related to the purported medicinal or cultural benefits derived from the animal parts $[62,63,74,79-$ 82 ], and this enduring relationship between food animals and medicinal therapy goes well beyond the understanding that adequate nutrition sustains a person's health. For instance, Werner [83] noted that much of the variation in the use or nonuse of lizards as food apparently stems from cultural beliefs concerning the medicinal or other benefits of their flesh. As an example of such cultural beliefs, in our study we found that some species used as food (e.g., Crassostrea rhizophorae, Anomalocardia brasiliana, and Eunectes murinus) were also considered to be aphrodisiacs.

Of the animals used as remedies and food, 52 (33.1\%) are under some form of legal protection, a result that clearly indicates the need for bringing all relevant stakeholders together to develop strategies that can more effectively deal with the issues related to the harvesting of wildlife for alimentary and/or medicinal purposes in Brazil. As discussed by Alves and Rosa [1], sustainability of harvesting of medicinal animals is challenged by many factors, from both social and ecological perspectives, and it is important to respect differing views of the value of wildlife, while, at the same time, conserving biodiversity.

Connections between traditional medicine, biodiversity, and human health have recently been addressed by different authors [84-88] and have drawn attention to the fact that biodiversity loss can have indirect and direct effects on human well-being as well. The reliance on traditional uses of animals as food and as medicine by communities around the world highlights the need for further interdisciplinary research in ethnozoology which can be used in strategies to conserve biodiversity.

\section{Acknowledgments}

The first and last authors would like to acknowledge Conselho Nacional de Desenvolvimento Científico e Tecnológico (CNPq) for providing a research fellowship. The authors also thanked the interviewees who contributed with the information to this research.

\section{References}

[1] R. R. N. Alves and I. L. Rosa, "Zootherapeutic practices among fishing communities in North and Northeast Brazil: a comparison," Journal of Ethnopharmacology, vol. 111, no. 1, pp. 82-103,
2007.

[2] A. Begossi and F. Braga, "Food taboos and folk medicine among fishermen from the Tocantins River," Amazoniana, vol. 12, pp. 341-352, 1992.

[3] R. R. N. Alves, I. L. Rosa, and G. G. Santana, "The role of animal-derived remedies as complementary medicine in Brazil," BioScience, vol. 57, no. 11, pp. 949-955, 2007.

[4] A. Begossi, "Food taboos at Búzios Island (SE Brazil): their significance and relation to folk medicine," Journal of Ethnobiology, vol. 12, no. 1, pp. 117-139, 1992.

[5] T. Johns, The Origins of Human Diet and Medicine, University of Arizona Press, Tucson, Arizona, 1996.

[6] T. Johns, With Bitter Herbs They Shall Eat It, University Arizona Press, Tucson, Arizona, 1990.

[7] N. L. Etkin and P. J. Ross, "Food as medicine and medicine as food. An adaptive framework for the interpretation of plant utilization among the Hausa of northern Nigeria," Social Science and Medicine, vol. 16, no. 17, pp. 1559-1573, 1982.

[8] N. L. Etkin, Eating on the Wild Side: The Pharmacologic, Ecologic and Social Implications of Using Noncultigens, University Arizona Press, Tucson, Arizona, 2000.

[9] A. Pieroni and L. L. Price, Eating and Healing: Traditional Food as Medicine, CRC Press, New York, NY, USA, 2006.

[10] N. L. Etkin, "Medicinal cuisines: diet and ethopharmacology," Pharmaceutical Biology, vol. 34, no. 5, pp. 313-326, 1996.

[11] L. Manniche, An Ancient Egyptian Herbal, University of Texas Press, Austin, Tex, USA, 1989.

[12] C. P. Bryan, Ancient Egyptian Medicine: The Papyrus Ebers, Ares, Chicago, Ill, USA, 1930.

[13] J. W. Estes, The Medical Skills of Ancient Egypt, Science History Publication, Canton, Mass, USA, 1989.

[14] C. Stetter, The Secret Medicine of the Pharaohs-Ancient Egyptian Healing, Edition Q, Chicago, Ill, USA, 1st edition, 1993.

[15] J. F. Nunn, Ancient Egyptian Medicine, University of Oklahoma Press, Norman, Okla, USA, 2002.

[16] L. C. Mackinney, "Animal substances in materia medica: a study in the persistence of the primitive," Journal of the History of Medicine and Allied Sciences, vol. 1, no. 1, pp. 149-170, 1946.

[17] R. C. Chemas, "A zooterapia no âmbito da medicina civilizada. I. Organoterapia humana e animal stricto sensu," in Zooterapia: Os Animais na Medicina Popular Brasileira, E. M. Costa-Neto and R. R. N. Alves, Eds., pp. 75-102, NUPEEA, Recife, Brazil, 2010.

[18] J. Chen, "Regulatory control of functional food in China," Scandinavian Journal of Nutrition/Naringsforskning, vol. 44, no. 3, pp. 130-131, 2000.

[19] W. Weng and J. Chen, "The Eastern perspective on functional foods based on traditional Chinese medicine," Nutrition Reviews, vol. 54, no. 11, pp. S11-S16, 1996.

[20] J. Farquhar, "Eating chinese medicine," Cultural Anthropology, vol. 9, no. 4, pp. 471-497, 1994.

[21] A. Fleuret, "Dietary and therapeutic uses of fruit in three Taita communities," in Forests, Trees and Food, FAO, Ed., Food and Agriculture Organization, Rome, Italy, 1993.

[22] G. Moreno-Black, P. Somnasang, and S. Thamathawan, "Cultivating continuity and creating change: women's home garden practices in Northeastern Thailand," Agriculture and Human Values, vol. 13, no. 3, pp. 3-11, 1996.

[23] A. Pieroni and A. Grazzini, "Alimenti-medicina di origine animale," in Herbs, Humans and Animals/Erbe, uomini e bestie, A. Pieroni, Ed., pp. 155-171, VDM, London, UK, 1999. 
[24] A. Pieroni, M. E. Giusti, and A. Grazzini, "Animal remedies in the folk medicinal practices of the Lucca and Pistoia Provinces, Central Italy," in Des sources du savoir aux médicaments du futur/From the Sources of Knowledge to the Medicines of the Future, J. Fleurentin, J. M. Pelt, and G. Mazars, Eds., pp. 371375, IRD Editions, Paris, France, 2002.

[25] R. R. N. Alves and I. L. Rosa, "From cnidarians to mammals: the use of animals as remedies in fishing communities in NE Brazil," Journal of Ethnopharmacology, vol. 107, no. 2, pp. 259-276, 2006.

[26] R. R. N. Alves and I. L. Rosa, "Zootherapy goes to town: the use of animal-based remedies in urban areas of NE and N Brazil," Journal of Ethnopharmacology, vol. 113, no. 3, pp. 541-555, 2007.

[27] R. R. N. Alves and I. L. Rosa, "Trade of animals used in Brazilian traditional medicine: trends and implications for conservation," Human Ecology, vol. 38, no. 5, pp. 691-704, 2010.

[28] F. S. Ferreira, N. L. G. Silva, E. F. F. Matias et al., "Potentiation of aminoglycoside antibiotic activity using the body fat from the snake Boa constrictor," Brazilian Journal of Pharmacognosy, vol. 21, no. 3, pp. 503-509, 2011.

[29] A. Begossi, "Food taboos-a scientific reason?" in Plants for Food and Medicine, H. D. V. Pendergast, N. Etkin, D. R. Harris, and P. J. Houghton, Eds., pp. 41-46, Royal Botanic Gardens, Kew, UK, 1998.

[30] FAO, Forests, Trees and Food, Food and Agriculture Organization, Rome, Italy, 1992.

[31] IIED, Whose Eden? An Overview of Community Approaches to Wildlife Management, Overseas Development Administration, London, UK, 1994.

[32] R. R. N. Alves, "Fauna used in popular medicine in Northeast Brazil," Journal of Ethnobiology and Ethnomedicine, vol. 5, article 1, 30 pages, 2009.

[33] E. Rodrigues, "Plants and animals utilized as medicines in the Jaú National Park (JNP), Brazilian Amazon," Phytotherapy Research, vol. 20, no. 5, pp. 378-391, 2006.

[34] U. P. Albuquerque and R. F. P. Lucena, "Métodos e técnicas para coleta de dados," in Métodos e Técnicas na Pesquisa Etnobotânica, U. P. Albuquerque and R. F. P. Lucena, Eds., pp. 37-62, NUPEEA/Livro Rápido, Recife, Brazil, 2004.

[35] K. Bailey, Methods of Social Research, Free Press, New York, NY, USA, 1994.

[36] H. P. Huntington, "Using traditional ecological knowledge in science: methods and applications," Ecological Applications, vol. 10, no. 5, pp. 1270-1274, 2000.

[37] A. B. M. Machado, G. M. Drummond, and A. P. Paglia, Livro Vermelho da fauna brasileira ameaçada de extinção, MMA, Fundação Biodiversitas, Brasília, Brasil, 2008.

[38] MMA, "Lista Nacional das espécies de invertebrados aquáticos e peixes sobreexplotadas ou ameaçadas de sobreexplotação. Instrução normativa 5, de 21 de maio de 2004," Diário Oficial da União, vol. 102, pp. 136-142, 2004.

[39] M. A. Huffman, "Self-medicative behavior in the African great apes: an evolutionary perspective into the origins of human traditional medicine," BioScience, vol. 51, no. 8, pp. 651-661, 2001.

[40] J. O’Hara-May, "Food or medicine," Transactions of the British Society for the History of Pharmacy, vol. 1, pp. 61-70, 1971.

[41] F. D. B. P. Moura and J. G. W. Marques, "Folk medicine using animals in the Chapada Diamantina: incidental medicine?" Ciencia e Saude Coletiva, vol. 13, no. 2, pp. 2179-2188, 2008.

[42] R. R. N. Alves and I. L. Rosa, Animals in Traditional Folk Medicine: Implications for Conservation, Springer, Berlin, Germany, 2013.
[43] J. Burger and M. Gochfeld, "Ocean ecosystems and human health," in Oceans and Human Health: Risks and Remedies from the Seas, P. J. Walsh, S. L. Smith, L. E. Fleming, H. Solo-Gabriele, and W. H. Gerwick, Eds., pp. 145-160, Academic Press, New York, NY, USA, 2008.

[44] C. Bouzan, J. T. Cohen, W. E. Connor et al., "A quantitative analysis of fish consumption and stroke risk," American Journal of Preventive Medicine, vol. 29, no. 4, pp. 347-352, 2005.

[45] J. T. Cohen, D. C. Bellinger, and B. A. Shaywitz, "A quantitative analysis of prenatal methyl mercury exposure and cognitive development," American Journal of Preventive Medicine, vol. 29, no. 4, p. 353, 2005.

[46] J. T. Cohen, D. C. Bellinger, W. E. Connor, and B. A. Shaywitz, "A quantitative analysis of prenatal intake of $n-3$ polyunsaturated fatty acids and cognitive development," American Journal of Preventive Medicine, vol. 29, no. 4, p. 366, 2005.

[47] M. Daviglus, J. Sheeshka, and E. Murkin, "Health benefits from eating fish," Comments on Toxicology, vol. 8, no. 4-6, pp. 345374, 2002.

[48] A. König, C. Bouzan, J. T. Cohen et al., "A quantitative analysis of fish consumption and coronary heart disease mortality," American Journal of Preventive Medicine, vol. 29, no. 4, pp. 335346, 2005.

[49] A. J. McMichael and C. D. Butler, "Fish, health, and sustainability," American Journal of Preventive Medicine, vol. 29, no. 4, pp. 322-323, 2005.

[50] J. Patterson, "Introduction-comparative dietary risk: balance the risk and benefits of fish consumption," Comments on Toxicology, vol. 8, no. 4-6, pp. 337-344, 2002.

[51] W. C. Willett, "Fish: balancing health risks and benefits," American Journal of Preventive Medicine, vol. 29, no. 4, pp. 320321, 2005.

[52] J. Burger and M. Gochfeld, "Perceptions of the risks and benefits of fish consumption: individual choices to reduce risk and increase health benefits," Environmental Research, vol. 109, no. 3, pp. 343-349, 2009.

[53] J. Ojasti, Wildlife Utilization in Latin America: Current Situation and Prospects for Sustainable Management, Food and Agriculture Organization of the United Nations, Rome, Italy, 1997.

[54] K. H. Redford and J. G. Robinson, "The game of choice: patterns of Indian and colonist hunting in the neotropics," American Anthropologist, vol. 89, no. 3, pp. 650-667, 1987.

[55] J. G. Robinson and K. H. Redford, Neotropical Wildlife Use and Conservation, University of Chicago Press, Chicago, Ill, USA, 1991.

[56] K. H. Redford and J. G. Robinson, "Subsistence and commercial uses of wildlife in Latin America," in Neotropical Wildlife Use and Conservation, J. G. Robinson and K. H. Redford, Eds., pp. 6-23, 1991.

[57] M. S. Alvard, J. G. Robinson, K. H. Redford, and H. Kaplan, "The sustainability of subsistence hunting in the neotropics," Conservation Biology, vol. 11, no. 4, pp. 977-982, 1997.

[58] N. Hanazaki, R. R. N. Alves, and A. Begossi, "Hunting and use of terrestrial fauna used by Caiçaras from the Atlantic Forest coast (Brazil)," Journal of Ethnobiology and Ethnomedicine, vol. 5, no. 1, article 36, 2009.

[59] J. A. A. Barbosa, V. A. Nobrega, and R. R. N. Alves, "Hunting practices in the semiarid region of Brazil," Indian Journal of Traditional Knowledge, vol. 10, no. 3, pp. 486-490, 2011. 
[60] R. R. N. Alves, L. E. T. Mendonça, M. V. A. Confessor, W. L. S. Vieira, and L. C. S. Lopez, "Hunting strategies used in the semiarid region of northeastern Brazil," Journal of Ethnobiology and Ethnomedicine, vol. 5, no. 12, article 56, 2009.

[61] L. R. Angeletti, U. Agrimi, C. Curia, D. French, and R. MarianiCostantini, "Healing rituals and sacred serpents," The Lancet, vol. 340 , no. 8813 , pp. 223-225, 1992.

[62] J. B. Thorbjarnarson, C. J. Lagueux, D. Bolze, M. W. Klemens, and A. B. Meylan, "Human use of turtle: a worldwide perspective," in Turtle Conservation, M. W. Klemens, Ed., pp. 33-84, Smithsonian Institution Press, London, UK, 2000.

[63] M. W. Klemens and J. B. Thorbjarnarson, "Reptiles as a food resource," Biodiversity and Conservation, vol. 4, no. 3, pp. 281298, 1995.

[64] R. R. N. Alves, N. A. Neto, G. G. Santana, W. L. S. Vieira, and W. O. Almeida, "Reptiles used for medicinal and magic religious purposes in Brazil," Applied Herpetology, vol. 6, no. 3, pp. 257274, 2009.

[65] R. R. D. Alves, W. L. D. Vieira, and G. G. Santana, "Reptiles used in traditional folk medicine: conservation implications," Biodiversity and Conservation, vol. 17, no. 8, pp. 2037-2049, 2008.

[66] R. R. N. Alves, K. S. Vieira, G. G. Santana et al., "A review on human attitudes towards reptiles in Brazil," Environmental Monitoring and Assessment, vol. 184, no. 11, pp. 6877-6901, 2012.

[67] R. R. N. Alves, G. A. Pereira Filho, K. Silva Vieira et al., "A zoological catalogue of hunted reptiles in the semiarid region of Brazil," Journal of Ethnobiology and Ethnomedicine, vol. 8, no. 1, pp. 1-27, 2012.

[68] H. Fernandes-Ferreira, R. L. Cruz, D. M. Borges-Nojosa, and R. R. N. Alves, "Crenças Associadas a Serpentes no Estado do Ceará, Nordeste do Brasil,” Sitientibus, vol. 11, no. 2, pp. 153-163, 2011.

[69] A. M. Rea, "Resource utilization and food taboos of Sonoran desert peoples," Journal of Ethnobiology, vol. 1, pp. 69-83, 1981.

[70] A. L. Silva, "Comida de gente: preferências e tabus alimentares entre os ribeirinhos do Médio Rio Negro (Amazonas, Brasil)," Revista de Antropologia, vol. 50, no. 1, pp. 125-179, 2007.

[71] R. R. N. Alves, G. G. Santana, and I. L. Rosa, "The role of animal-derived remedies as complementary medicine in Brazil," in Animals in Traditional Folk Medicine: Implications for Conservation, R. R. N. Alves and I. L. Rosa, Eds., pp. 289-301, Springer, Berlin, Germany, 2013.

[72] R. R. N. Alves, "Relationships between fauna and people and the role of ethnozoology in animal conservation," Ethnobiology and Conservation, vol. 1, pp. 1-69, 2012.

[73] E. L. Bennett and J. G. Robinson, "Hunting for the Snark," in Hunting for Sustainability in Tropical Forests, J. G. Robinson and E. L. Bennett, Eds., pp. 1-9, Columbia University Press, New York, NY, USA, 2000.

[74] E. L. Bennett and J. G. Robinson, "Hunting of wildlife in tropical forests," The World Bank Environment Department Papers, vol. 76, pp. 1-42, 2000.

[75] R. Nasi, D. Brown, D. Wilkie et al., Conservation and Use of Wildlife-Based Resources: The Bushmeat Crisis, Secretariat of the Convention on Biological Diversity and Center for International Forestry Research (CIFOR), Bogor, Indonesia, 1st edition, 2008.

[76] W. R. Townsend, "The sustainability of subsistence hunting by the Sirionó Indians of Bolivia," in Hunting for Sustainability in Tropical Forests, J. G. Robinson and E. L. Bennett, Eds., pp. 267281, Columbia University Press, New York, NY, USA, 2000.
[77] "MMA Status of the National Biodiversity Strategy Advances," in Proceedings of the 2003 Meeting for Identification of Issues on Biodiversity for Cooperation and Interchanging among South American countries, Rio de Janeiro, Brazil, December 2003.

[78] C. Seixas and A. Begossi, "Ethnozoology of caiçaras from Aventureiro, Ilha Grande," Journal of Ethnobiology, vol. 21, no. 1, pp. 107-135, 2001.

[79] H. S. Fitch, R. W. Henderson, and D. M. Hillis, "Exploitation of iguanas in Central America," in Iguanas of the World Their Behavior, Ecology and Conservation, pp. 397-415, Noyes, Saddle River, NJ, USA, 1982.

[80] R. R. N. Alves, W. M. S. Souto, and R. R. D. Barboza, "Primates in traditional folk medicine: a world overview," Mammal Review, vol. 40, no. 2, pp. 155-180, 2010.

[81] R. R. N. Alves, R. R. D. Barboza, and W. M. S. Souto, "A Global overview of canids used in traditional medicines," Biodiversity and Conservation, vol. 19, no. 6, pp. 1513-1522, 2010.

[82] H. Hilaluddin, R. Kaul, and D. Ghose, "Conservation implications of wild animal biomass extractions in Northeast India," Animal Biodiversity and Conservation, vol. 28, no. 2, pp. 169179, 2005.

[83] D. I. Werner, "The rational use of green iguanas," in Neotropical Wildife Use and Conservation, J. G. Robinson and K. H. Redford, Eds., pp. 181-201, The University of Chicago Press, Chicago, Ill, USA, 1991.

[84] R. R. N. Alves and I. L. Rosa, "Biodiversity, traditional medicine and public health: where do they meet?" Journal of Ethnobiology and Ethnomedicine, vol. 3, article 14, 9 pages, 2007.

[85] C. Anyinam, "Ecology and ethnomedicine: exploring links between current environmental crisis and indigenous medical practices," Social Science and Medicine, vol. 40, no. 3, pp. 321329, 1995.

[86] A. J. McMichael and R. Beaglehole, "The changing global context of public health," The Lancet, vol. 356, no. 9228, pp. 495499, 2000.

[87] E. Chivian, Biodiversity: Its Importance to Human Health, Center for Health and the Global Environment. Harvard Medical School Cambridge, Cambridge, Mass, USA, 2002.

[88] R. R. N. Alves and I. L. Rosa, "Why study the use of animal products in traditional medicines?" Journal of Ethnobiology and Ethnomedicine, vol. 1, article 5, 5 pages, 2005. 


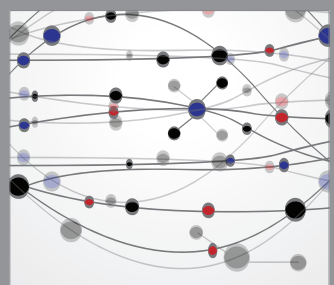

The Scientific World Journal
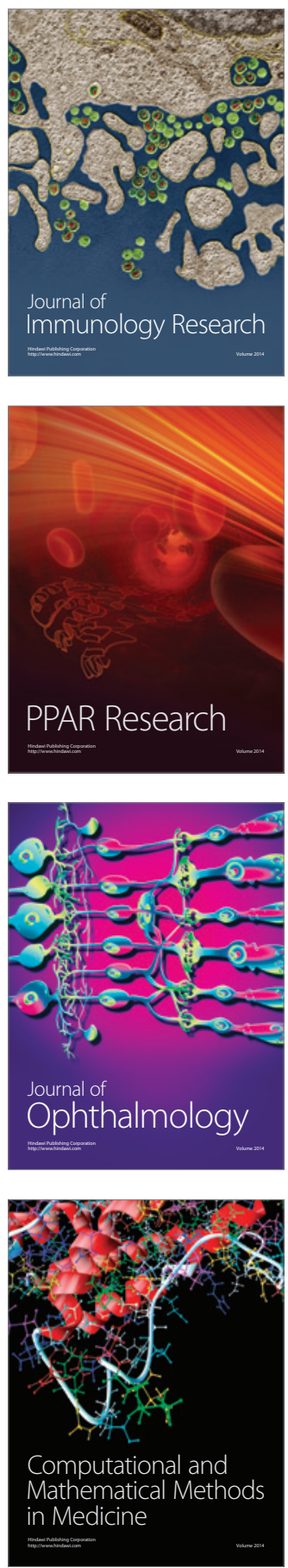

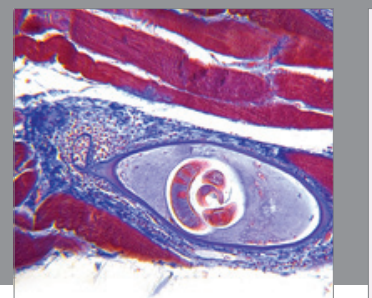

Gastroenterology

Research and Practice
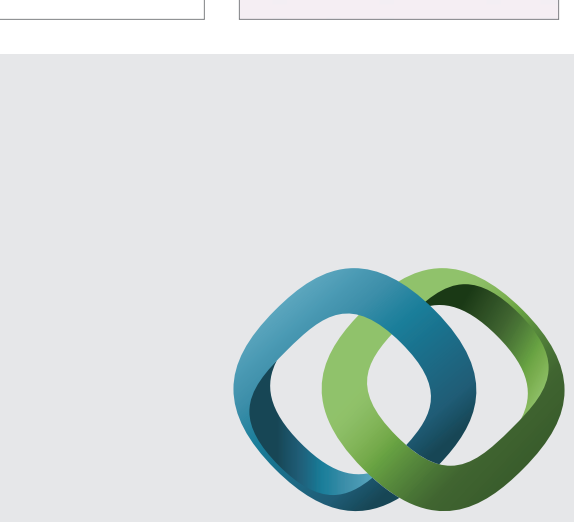

\section{Hindawi}

Submit your manuscripts at

http://www.hindawi.com
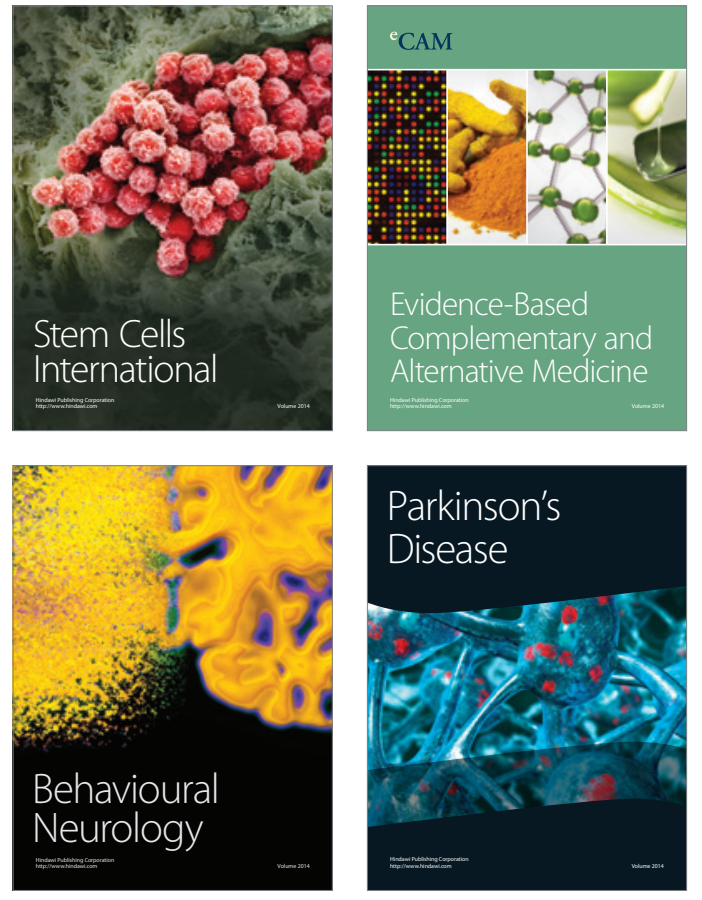
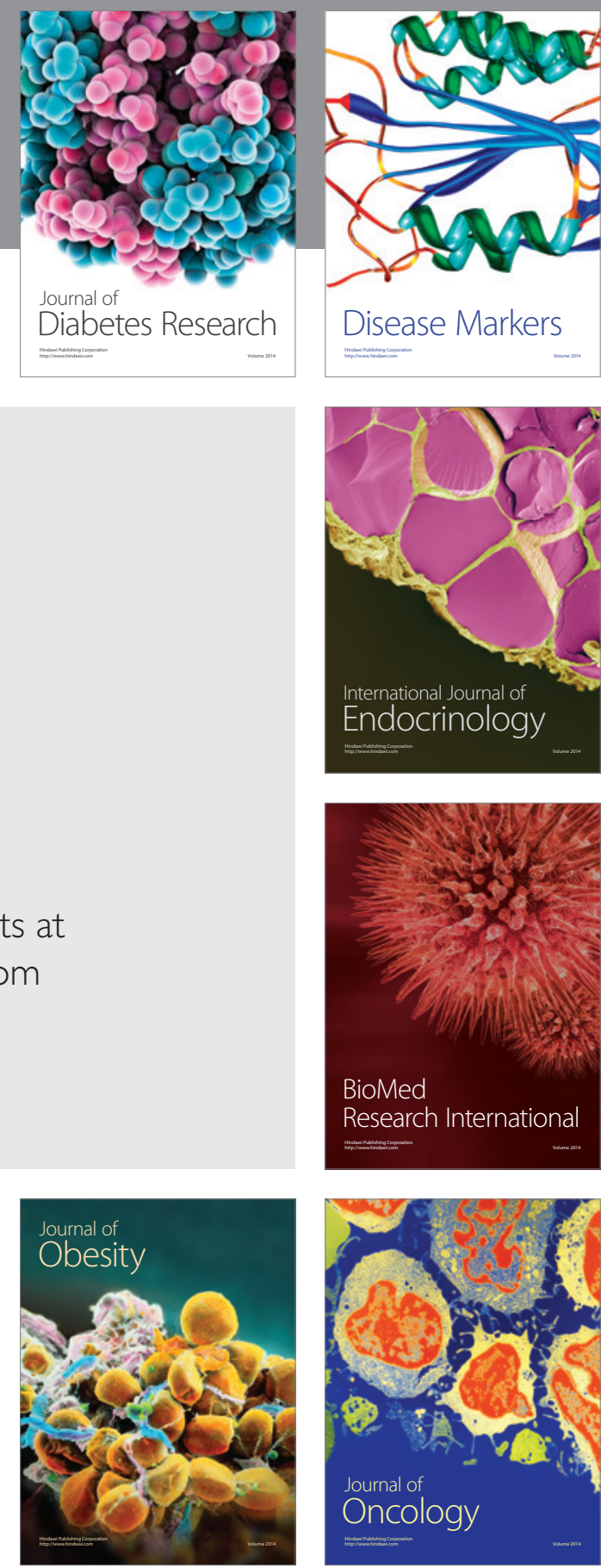

Disease Markers
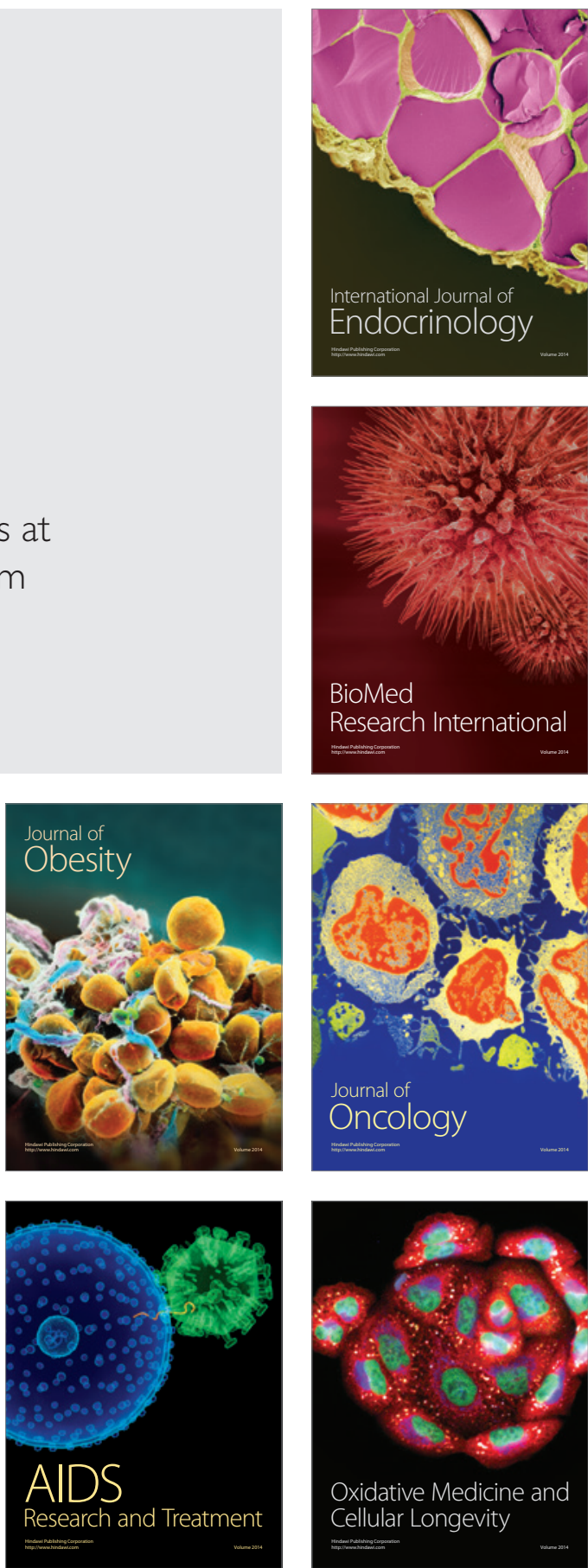